\title{
Control and Synchronization of New Hyperchaotic System using Active Backstepping Design
}

\author{
Sung-Hun Yu* , Chang-Ho Hyun ${ }^{* *+}$ and Mignon Park ${ }^{*+}$ \\ * School of Electrical and Electronic Engineering, Yonsei University \\ ** Division of Electrical Electronic and Control Engineering, Kongju National University
}

\begin{abstract}
In this paper, an active backstepping design is proposed to achieve control and synchronization of a new hyperchaotic system. The proposed method is a systematic design approach and exists in a recursive procedure that interlaces the choice of a Lyapunov function with the design of the active control. The proposed controller enables stabilization of chaotic motion to the origin as well as synchronization of the two identical new hyperchaotic systems. Numerical simulations illustrate the validity of the proposed control technique.
\end{abstract}

Key Words : hyperchaotic system, chaos control, chaos synchronization, active control, backstepping control.

\section{Introduction}

Since the pioneering work by Ott, Grebogi and Yorke (OGY) [1] and Pecora and Carroll [2], chaos control and synchronization, as an important topic in the nonlinear science, has been widely investigated in a variety of fields, such as engineerings, physics, mathematics, life sciences, biomedical communities, heart beat regulations, etc.

It is well known that for regular chaotic systems, there is just one positive Lyapunov exponent. Messages masked by regular chaotic systems are not always safe [3]. It was suggested that this problem can be overcome by using higher-dimensional hyperchaotic systems, which have increased randomness and unpredictability [4]. The hyperchaotic attractor is characterized as a chaotic attractor with more than one positive Lyapunov exponent, and indicates that the dynamics of the system are expanded in more than one direction. Due to its higher unpredictability than regular chaotic systems, hyperchaos may be more useful in some relevant applications. Therefore, how to realize control and synchronization of hyperchaotic systems is an interesting and challenging work.

Until now, enormous progresses have been made in understanding various methods [5-17] to achieve control and synchronization of chaotic systems. Fortunately, the existing method to control and synchronize chaotic systems can be generalized to control and synchronize hyperchaotic systems [18-20]. Among the aforementioned method, the active control [9-13] and the backstepping design [14-17] have been widely recognized as two powerful design methods for control and synchronization of chaotic systems. Chaos synchronization

Manuscript received Mar. 16, 2011; revised Apr. 25, 2011; +Corresponding Authors

This research project was supported by the Sports Promotion Fund of Seoul Olympic Sports Promotion Foundation from Ministry of Culture, Sports and Tourism. using the active control was proposed by Bai and Lonngren for the Lorenz system [9]. The Active control technique can be used widely to control various nonlinear systems including chaotic systems since it has the flexiblity to design a control law. The backstepping design method can guarantee the global stability, and the tracking and transient performance for a broad class of strict-feedback nonlinear systems [21].The technique is a systematic design approach and consists in a recursive procedure that skillfully interlaces the choice of a Lyapunov function with the control. Consequently, the main aim of this paper is in an attempt to use the combination of the two control approaches, i.e., active backstepping method, to control and synchronize hyperchaotic system.

In this paper, the active backstepping control scheme is proposed to control and synchronize a new hyperchaotic system that is recently presented by Qi at al. [22]. The new hyperchaotic system has two large positive and one small negative Lyapunov exponents over the large range of parameters. Spectral analysis shows that the system in the hyperchaotic mode has an extremely broad frequency bandwidth of high magnitudes, verifying its unusual random nature and indicating its great potential for some pertinent engineering applications [23-24].

The rest of the paper is organized as follow. In Section 2, a brief description of the new hyperchaotic system is introduced. We present the control scheme of hyperchaotic system in Section 3. Section 4 deals with the synchronization behavior of two identical new hyperchaotic systems and finally some concluding remarks are made in Section 5.

\section{System Description}

The new 4D hyperchaotic system was performed by Qi et al. [22]. This chaotic system, named as Qi system in this paper, is described by the following nonlinear differential equations. 


$$
\begin{aligned}
& \dot{x}_{1}=a\left(x_{2}-x_{1}\right)+x_{2} x_{3} \\
& \dot{x}_{2}=b\left(x_{1}+x_{2}\right)-x_{1} x_{3} \\
& \dot{x}_{3}=-c x_{3}-g x_{4}+x_{1} x_{2} \\
& \dot{x}_{4}=-f x_{4}+h x_{3}+x_{1} x_{2}
\end{aligned}
$$

where $x_{1}, x_{2}, x_{3}$ and $x_{4}$ are state variables and $a, b$, $c, f, g$ and $h$ are all positive real constant parameters. When $a=50, b=24, c=13, f=8, g=33$ and $h=30$, the system (1) is hyperchaotic, and the hyperchaotic attractors are shown in Fig. 1.

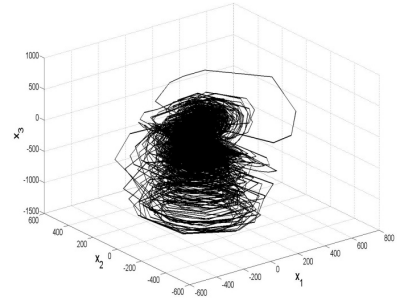

(a) $x_{1}-x_{2}-x_{3}$

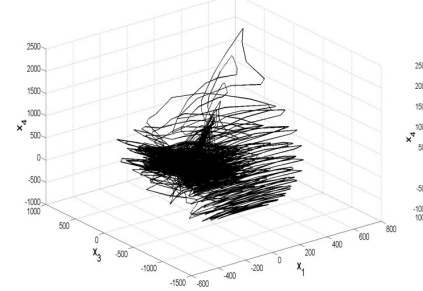

(c) $x_{1}-x_{3}-x_{4}$

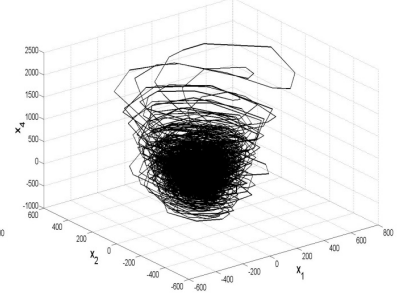

(b) $x_{1}-x_{2}-x_{4}$

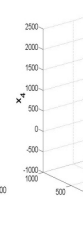

(d) $x_{2}-x_{3}-x_{4}$
Fig 1. 3-D Phase portrait of the hyperchaotic system (1)

\section{Control of the New Hyperchaotic System}

\subsection{Active Backstepping Control}

In the following, we will use the backstepping design method to design an active controller for the hyperchaotic system presented by the equation (1) to the origin. According to the active control theory, the controlled hyperchaotic system can be written in the following form

$$
\begin{aligned}
& \dot{x}_{1}=a\left(x_{2}-x_{1}\right)+x_{2} x_{3}+u_{1} \\
& \dot{x}_{2}=b\left(x_{1}+x_{2}\right)-x_{1} x_{3}+u_{2} \\
& \dot{x}_{3}=-c x_{3}-g x_{4}+x_{1} x_{2}+u_{3} \\
& \dot{x}_{4}=-f x_{4}+h x_{3}+x_{1} x_{2}+u_{4}
\end{aligned}
$$

where $u=\left[u_{1}, u_{2}, u_{3}, u_{4}\right]^{T}$ is the active control function.

In practical applications, the controller to be designed must be simple, efficient and easy to implement. Thus, let $u_{1}=0$ and $u_{4}=0$, then the controlled dynamics can be written as

$$
\begin{aligned}
& \dot{x}_{1}=a\left(x_{2}-x_{1}\right)+x_{2} x_{3} \\
& \dot{x}_{2}=b\left(x_{1}+x_{2}\right)-x_{1} x_{3}+u_{2} \\
& \dot{x}_{3}=-c x_{3}-g x_{4}+x_{1} x_{2}+u_{3} \\
& \dot{x}_{4}=-f x_{4}+h x_{3}+x_{1} x_{2}
\end{aligned}
$$

Now our objective is to find $u_{2}$ and $u_{3}$ that make the state vectors $x_{i}=\left[x_{1}, x_{2}, x_{3}, x_{4}\right]^{T}$ converge to zero as time $t$ goes to infinity. In order to achieve such goal, the backstepping design method is adopted.

The backstepping design procedure is recursive. At the $i$ thstep, the $i$ th-order subsystem is stabilized with respect to a Lyapunov function $V_{i}$ by the design of a virtual control $\alpha_{i}$ and the control input function $u_{i}$. Now we begin to design the active controller based on the backstepping design method as follows.

Step 1. Let $z_{1}=x_{1}$, then we can obtain its derivative as follows

$$
\begin{aligned}
\dot{z}_{1} & =\dot{x}_{1} \\
& =-a z_{1}+\left(a+x_{3}\right) x_{2}
\end{aligned}
$$

where $x_{2}=\alpha_{1}\left(z_{1}\right)$ is regarded as a virtual control input.

For the design of $\alpha_{1}$ to stabilize $z_{1}$-subsystem (4), we choose Lyapunov function $V_{1}$ as

$$
V_{1}=\frac{z_{1}^{2}}{2}
$$

The derivative of $V_{1}$ is obtained as

$$
\begin{aligned}
\dot{V}_{1} & =z_{1} \dot{z}_{1} \\
& =-a z_{1}^{2}+\left(a+x_{3}\right) z_{1} \alpha_{1}
\end{aligned}
$$

If we choose $\alpha_{1}=0$, then $\dot{V}_{1}$ is negative definite. This implies that the $z_{1}$-subsystem (4) is asymptotically stable.

Since the virtual control function $\alpha_{1}$ is estimative, the error between $x_{2}$ and $\alpha_{1}$ is

$$
z_{2}=x_{2}-\alpha_{1}
$$

Then, we can obtain the following $\left(z_{1}, z_{2}\right)$-subsystem

$$
\begin{aligned}
& \dot{z}_{1}=-a z_{1}+a z_{2}+z_{2} x_{3} \\
& \dot{z}_{2}=b z_{1}+b z_{2}-z_{1} x_{3}+u_{2}
\end{aligned}
$$

where $x_{3}=\alpha_{2}\left(z_{1}, z_{2}\right)$ is regarded as a virtual controller.

Step 2. In this step, we stabilize the $\left(z_{1}, z_{2}\right)$-subsystem (8). We can choose a Lyapunov function $V_{2}$ as follows

$$
V_{2}=V_{1}+\frac{z_{2}^{2}}{2}
$$

Its derivative is given by

$$
\begin{aligned}
\dot{V}_{2} & =\dot{V}_{1}+z_{2} \dot{z}_{2} \\
& =-a z_{1}^{2}+z_{2}\left\{(a+b) z_{1}+b z_{2}+u_{2}\right\}
\end{aligned}
$$

If we choose $\alpha_{2}=0$ and $u_{2}=-(a+b) z_{1}-2 b z_{2}$,

then $\dot{V}_{2}=-a z_{1}^{2}-b z_{2}^{2}<0$ makes $\left(z_{1}, z_{2}\right)$-subsystem asymptotically stable. Similarly, assume that $z_{3}=x_{3}-\alpha_{2}$, then we can derive the following $\left(z_{1}, z_{2}, z_{3}\right)$-subsystem 


$$
\begin{aligned}
& \dot{z}_{1}=-a z_{1}+a z_{2}+z_{2} z_{3} \\
& \dot{z}_{2}=-a z_{1}-b z_{2}-z_{1} z_{3} \\
& \dot{z}_{3}=-c z_{3}-g x_{4}+z_{1} z_{2}+u_{3}
\end{aligned}
$$

Step 3. In order to stabilize the $\left(z_{1}, z_{2}, z_{3}\right)$-subsystem (11), we can choose a Lyapunov function $V_{3}$ as follows

$$
V_{3}=V_{2}+\frac{z_{3}^{2}}{2}
$$

Its derivative of $V_{3}$ is

$$
\begin{aligned}
\dot{V}_{3} & =\dot{V}_{2}+z_{3} \dot{z}_{3} \\
& =-a z_{1}^{2}-b z_{2}^{2}+z_{3}\left(-c z_{3}-g x_{4}+z_{1} z_{2}+u_{3}\right)
\end{aligned}
$$

If we choose $u_{3}=g x_{4}-z_{1} z_{2}$,

then $\dot{V}_{3}=-a z_{1}^{2}-b z_{2}^{2}-c z_{3}^{2}<0$ makes the $\left(z_{1}, z_{2}, z_{3}\right)$ subsystem (11) asymptotically stable

Since $\dot{V}_{3}$ is negative definite, it follows that the equilibrium $(0,0,0)$ of the subsystem (11) is global asymptotically stable. Furthermore, since $z_{1}=x_{1}, \quad z_{2}=x_{2}-\alpha_{1}=x_{2} \quad$ and $z_{3}=x_{3}-\alpha_{2}=x_{3}, x_{1}, x_{2}$ and $x_{3}$ go to zeros asymptotically as well. According to $x_{1} \rightarrow 0, x_{2} \rightarrow 0, x_{3} \rightarrow 0$ and the fourth equation of system (3), we get that $\left(x_{1}, x_{2}, x_{3}, x_{4}\right)$ in the controlled system (3) tend to $(0,0,0,0)$ as $t \rightarrow \infty$. In other words, the controlled system (3) is asymptotically stable with the proposed control inputs.

\subsection{Numerical Results}

For the purpose of numerical simulation, we set $a=50$, $b=24, c=13, f=8, g=33$ and $h=30$, as in Fig. 1, to ensure hyperchaotic behaviors. The initial conditions for the hyperchaotic system (3) set to be $x_{1}(0)=0.1, x_{2}(0)=0.2$, $x_{3}(0)=0.3, x_{4}(0)=0.4$. Fig. 2 shows the time response of states with the proposed control functions. The controller is added to the hyperchaotic system (3) at $t=20$. As expected, it shows that the hyperchotic system can be stabilized to the origin point $(0,0,0,0)$.

\section{Synchronization of New Hyperchaotic System}

\subsection{Active Backstepping Synchronization}

In this section, we will use the backstepping method to design an active controller to synchronize two identical Qi hyperchaotic systems.

In order to observe the synchronization behavior in the Qi hyperchaotic system, we assume the drive system as

$$
\begin{aligned}
& \dot{x}_{1}=a\left(x_{2}-x_{1}\right)+x_{2} x_{3} \\
& \dot{x}_{2}=b\left(x_{1}+x_{2}\right)-x_{1} x_{3} \\
& \dot{x}_{3}=-c x_{3}-g x_{4}+x_{1} x_{2} \\
& \dot{x}_{4}=-f x_{4}+h x_{3}+x_{1} x_{2}
\end{aligned}
$$

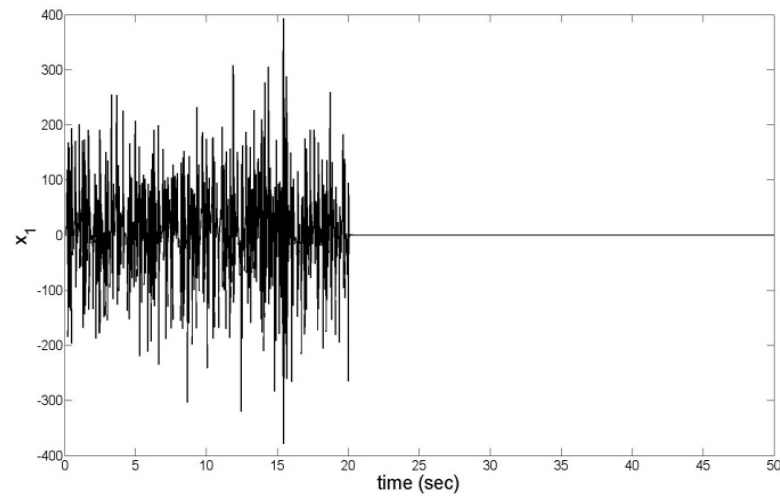

(a) The time response of states $x_{1}$

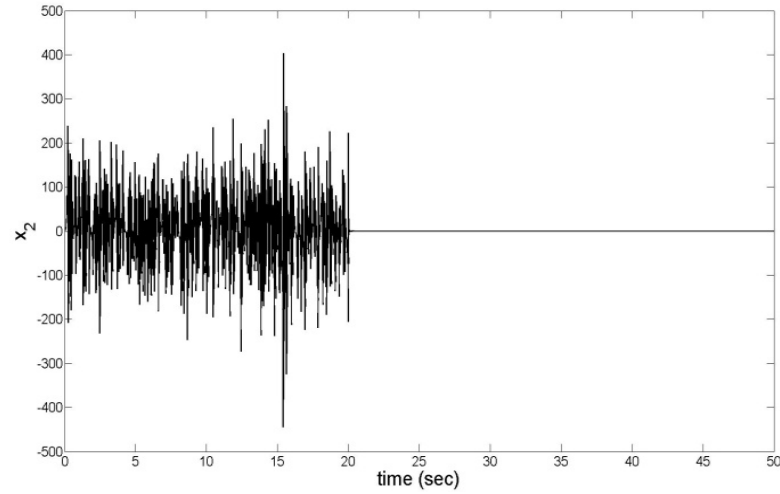

(b) The time response of states $x_{2}$

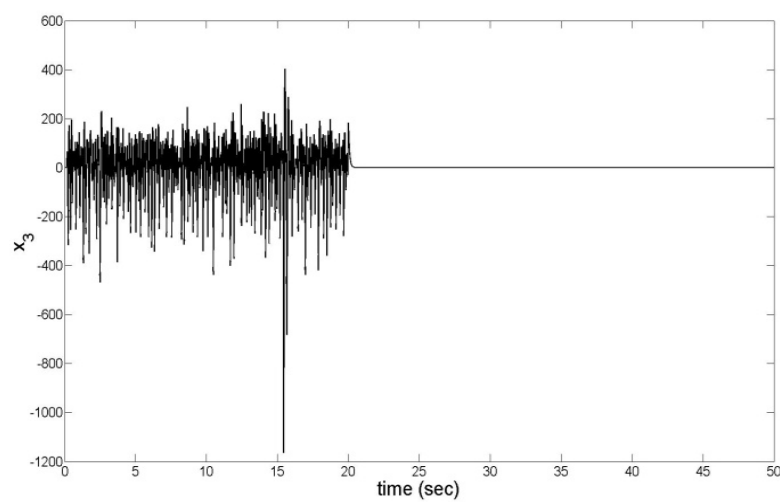

(c) The time response of states $x_{3}$

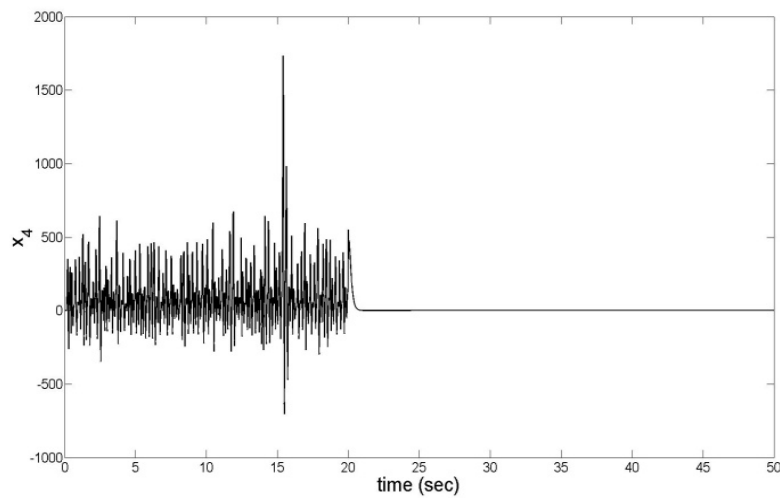

(d) The time response of states $x_{4}$

Fig. 2. The time response of states for the system (3) with the proposed controller. 
and the response system is

$$
\begin{aligned}
& \dot{y}_{1}=a\left(y_{2}-y_{1}\right)+y_{2} y_{3}+u_{1} \\
& \dot{y}_{2}=b\left(y_{1}+y_{2}\right)-y_{1} y_{3}+u_{2} \\
& \dot{y}_{3}=-c y_{3}-g y_{4}+y_{1} y_{2}+u_{3} \\
& \dot{y}_{4}=-f y_{4}+h y_{3}+y_{1} y_{2}+u_{4}
\end{aligned}
$$

where $u=\left[u_{1}, u_{2}, u_{3}, u_{4}\right]^{T}$ is the active control function. Likewise in section 2, let $u_{1}=0$ and $u_{4}=0$, then the response system dynamics can be written as

$$
\begin{aligned}
& \dot{y}_{1}=a\left(y_{2}-y_{1}\right)+y_{2} y_{3} \\
& \dot{y}_{2}=b\left(y_{1}+y_{2}\right)-y_{1} y_{3}+u_{2} \\
& \dot{y}_{3}=-c y_{3}-g y_{4}+y_{1} y_{2}+u_{3} \\
& \dot{y}_{4}=-f y_{4}+h y_{3}+y_{1} y_{2}
\end{aligned}
$$

Here, we aim at determining the controllers $u_{2}$ and $u_{3}$ which are required for the controlled response system (16) to synchronize with the drive system (14). For this purpose, let the error states between the state variables of the response system (16) and the drive system (14) be

$$
e_{1}=y_{1}-x_{1}, e_{2}=y_{2}-x_{2}, e_{3}=y_{3}-x_{3}, e_{4}=y_{4}-x_{4}
$$

Subtracting (14) from (16), we obtain the following error dynamics

$$
\begin{aligned}
& \dot{e}_{1}=a\left(e_{2}-e_{1}\right)+e_{2} e_{3}+x_{3} e_{2}+x_{2} e_{3} \\
& \dot{e}_{2}=b\left(e_{1}+e_{2}\right)-e_{1} e_{3}-x_{3} e_{1}-x_{1} e_{3}+u_{2} \\
& \dot{e}_{3}=-c e_{3}-g e_{4}+e_{1} e_{2}+x_{2} e_{1}+x_{1} e_{2}+u_{3} \\
& \dot{e}_{4}=-f e_{4}+h e_{3}+e_{1} e_{2}+x_{2} e_{1}+x_{1} e_{2}
\end{aligned}
$$

The next step is to find $u_{2}$ and $u_{3}$ that make the error vectors $e=\left[e_{1}, e_{2}, e_{3}, e_{4}\right]^{T}$ converge to zero as time $t$ goes to infinity. This implies that the trajectory of the response system (16) asymptotically approaches the trajectory of the drive system (14).

Again, we design the active controller based on the backstepping method outlined in subsection 3.1

Step 1. Let $z_{1}=e_{1}$, then we can obtain its derivative

$$
\begin{aligned}
\dot{z}_{1} & =\dot{e}_{1} \\
& =-a z_{1}+\left(a+x_{3}+e_{3}\right) e_{2}+x_{2} e_{3}
\end{aligned}
$$

where $e_{2}=\alpha_{1}\left(z_{1}\right)$ is regarded as a virtual control input.

For the design of $\alpha_{1}$ to stabilize $z_{1}$-subsystem (19), we choose Lyapunov function $V_{1}$ as

$$
V_{1}=\frac{z_{1}^{2}}{2} \text {. }
$$

The derivative of $V_{1}$ is as following

$$
\begin{aligned}
\dot{V}_{1} & =z_{1} \dot{z}_{1} \\
& =-a z_{1}^{2}+x_{2} z_{1} e_{3}+\left(a+x_{3}+e_{3}\right) z_{1} \alpha_{1}
\end{aligned}
$$

If we choose $\alpha_{1}=0$, then $\dot{V}_{1}=-a z_{1}^{2}+x_{2} z_{1} e_{3}$. The second term $x_{2} z_{1} e_{3}$ in $\dot{V}_{1}$ will be cancelled at the next step.

Since the virtual control input $\alpha_{1}$ is estimative the error between $e_{2}$ and $\alpha_{1}$ is

$$
z_{2}=e_{2}-\alpha_{1}
$$

Then, we can obtain the following $\left(z_{1}, z_{2}\right)$-subsystem;

$$
\begin{aligned}
& \dot{z}_{1}=-a z_{1}+\left(a+x_{3}\right) z_{2}+x_{2} e_{3}+z_{2} e_{3} \\
& \dot{z}_{2}=\left(b-x_{3}\right) z_{1}+b z_{2}-\left(x_{1}+z_{1}\right) e_{3}+u_{2}
\end{aligned}
$$

where $e_{3}=\alpha_{2}\left(z_{1}, z_{2}\right)$ is regarded as a virtual controller.

Step 2. In this step, we will stabilize the $\left(z_{1}, z_{2}\right)$-subsystem (23). We can choose a Lyapunov function $V_{2}$ as follows

$$
V_{2}=V_{1}+\frac{z_{2}^{2}}{2}
$$

Its derivative is given by

$$
\begin{aligned}
\dot{V}_{2}= & \dot{V}_{1}+z_{2} \dot{z}_{2} \\
= & -a z_{1}^{2}+\left(a+x_{3}+\alpha_{2}\right) z_{1} z_{2}+x_{2} z_{1} \alpha_{2} \\
& +z_{2}\left\{\left(b-x_{3}\right) z_{1}+b z_{2}-\left(x_{1}+z_{1}\right) \alpha_{2}+u_{2}\right\} \\
= & -a z_{1}^{2}+\left(x_{2} z_{1}-x_{1} z_{2}\right) \alpha_{2} \\
& +z_{2}\left\{(a+b) z_{1}+b z_{2}+u_{2}\right\}
\end{aligned}
$$

If we choose $\alpha_{2}=0$ and $u_{2}=-(a+b) z_{1}-2 b z_{2}$, then $\dot{V}_{2}=-a z_{1}^{2}-b z_{2}^{2}<0 \quad$ makes $\quad\left(z_{1}, z_{2}\right) \quad$-subsystem asymptotically stable. Similarly, assume that $z_{3}=e_{3}-\alpha_{2}$, then we can derive the following $\left(z_{1}, z_{2}, z_{3}\right)$-subsystem

$$
\begin{aligned}
& \dot{z}_{1}=-a z_{1}+\left(a+x_{3}\right) z_{2}+x_{2} z_{3}+z_{2} z_{3} \\
& \dot{z}_{2}=-\left(a+x_{3}\right) z_{1}-b z_{2}-x_{1} z_{3}-z_{1} z_{3} \\
& \dot{z}_{3}=x_{2} z_{1}+x_{1} z_{2}-c z_{3}-g e_{3}+z_{1} z_{2}+u_{3}
\end{aligned}
$$

Step 3. In order to stabilize the $\left(z_{1}, z_{2}, z_{3}\right)$-subsystem (26), we can choose a Lyapunov function $V_{3}$ as follows

$$
V_{3}=V_{2}+\frac{z_{3}^{2}}{2}
$$

Its derivative of $V_{3}$ is

$$
\begin{aligned}
\dot{V}_{3}= & \dot{V}_{2}+z_{3} \dot{z}_{3} \\
= & -a z_{1}^{2}-b z_{2}^{2} \\
& +z_{3}\left(2 x_{2} z_{1}-c z_{3}-g e_{4}+z_{1} z_{2}+u_{3}\right)
\end{aligned}
$$

If we choose $u_{3}=-2 x_{2} z_{1}+g e_{4}-z_{1} z_{2}$, then $\quad \dot{V}_{3}=-a z_{1}^{2}-b z_{2}^{2}-c z_{3}^{2}<0$ makes the $\left(z_{1}, z_{2}, z_{3}\right)$ subsystem (26) asymptotically stable.

Likewise, since $\dot{V}_{3}$ is negative definite, it follows that in the $\left(z_{1}, z_{2}, z_{3}\right)$ coordinates the equilibrium $(0,0,0)$ of the 
subsystem (26) is global asymptotically stable. In view of $z_{1}=e_{1}, \quad z_{2}=e_{2}-\alpha_{1}=e_{2}$ and $z_{3}=e_{3}-\alpha_{2}=e_{3}$, this implies that $e_{1}, e_{2}$ and $e_{3}$ go to zero asymptotically. According to $e_{1} \rightarrow 0, e_{2} \rightarrow 0, e_{3} \rightarrow 0$ and the fourth equation of system (18), we get that $\left(e_{1}, e_{2}, e_{3}, e_{4}\right)$ of the controlled system (18) go to $(0,0,0,0)$ as $t \rightarrow \infty$. In other words, the trajectory of the controlled response system (16) asymptotically approaches the trajectory of the drive system (14) with the proposed control inputs.

\subsection{Numerical Results}

Similarly, we set $a=50, b=24, c=13, f=8, g=33$ and $h=30$ to ensure hyperchaotic behavior. The initial conditions for the drive hyperchaotic system (14) and the response hyperchaotic system (16) are

$x_{1}(0)=0.1, x_{2}(0)=0.2, x_{3}(0)=0.3, x_{4}(0)=0.4$
$y_{1}(0)=1.0, y_{2}(0)=2.0, y_{3}(0)=3.0, y_{4}(0)=4.0$.

and

Thus, the initial values of the error states are $e_{1}(0)=0.9, e_{2}(0)=1.8 e_{3}(0)=2.7, e_{4}(0)=3.6$.

Fig. 3 shows the time response of states determined by the drive system and the response system with the proposed control function. The trajectories of synchronization errors for the drive system and the response system are shown in Fig. 4. The controller turns on at $t=3$. As expected, it shows that all state variables are synchronized and the synchronization errors converge to zero.

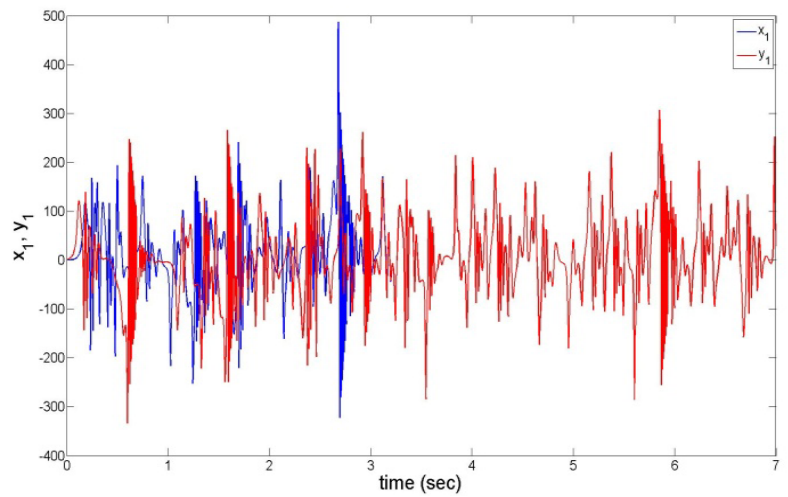

(a) The time response of states $x_{1}$ and $y_{1}$

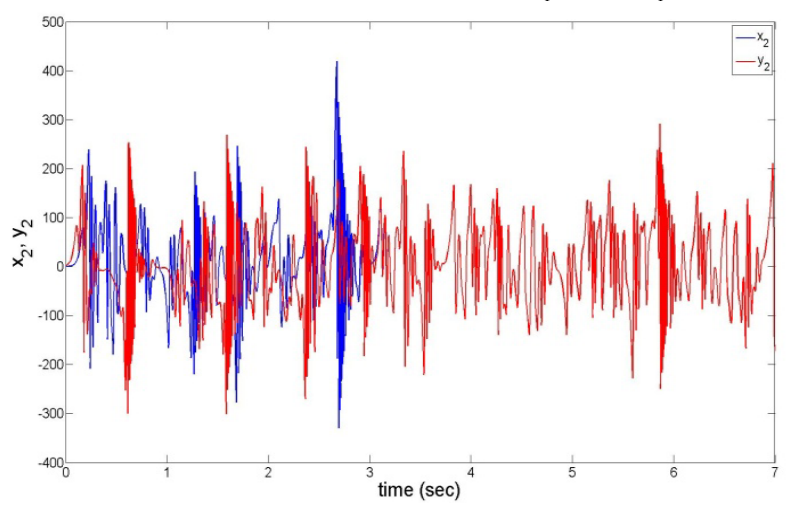

(b) The time response of states $x_{2}$ and $y_{2}$

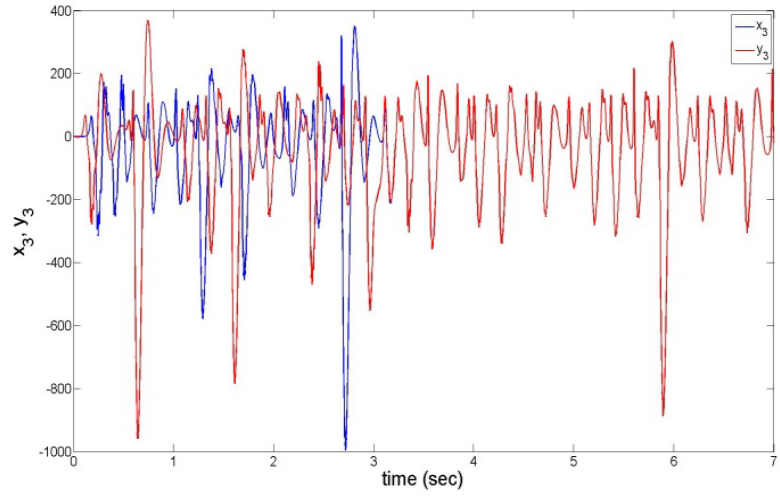

(c) The time response of states $x_{3}$ and $y_{3}$

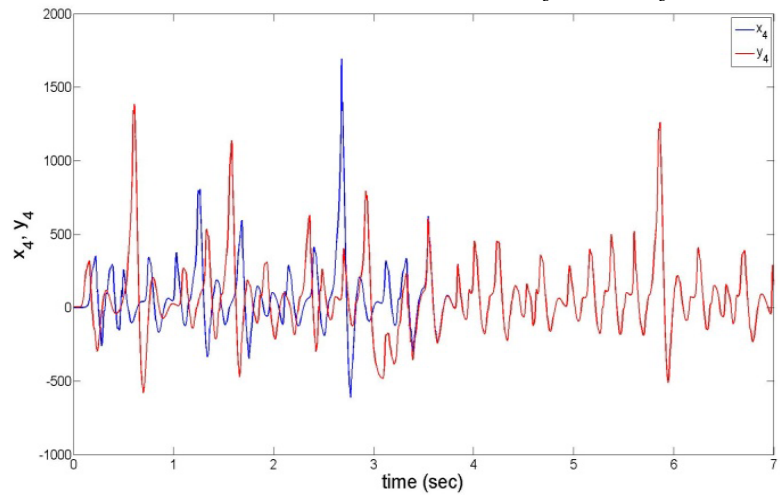

(d) The time response of states $x_{4}$ and $y_{4}$

Fig. 3. The time response of states for the drive system (14) and the response system (16) with the proposed controller.

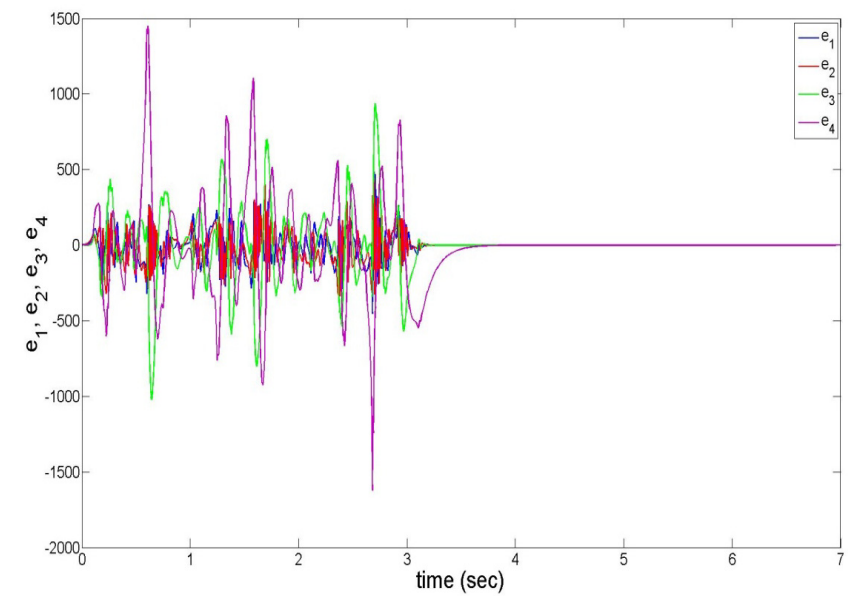

Fig. 4. The time response of synchronization error states

\section{Conclusions}

This paper has examined control and synchronization of the new hyperchaotic systems performed by Qi et al. [22] using the active backstepping control method. The proposed scheme is a systematic design approach and consists in a recursive procedure that interlaces the choice of a Lyapunov function with the design of active control. The proposed control approach is able to stabilize the chaotic motion to the origin. In addition, it 
synchronizes the two identical Qi hyperchaotic systems with the systematic way. Numerical simulations were also carried out to illustrate the effectiveness of the approach. We verified that the proposed method has following advantages. First, it does not need to calculate the Lyapunov exponents and eigenvalues of the Jacobian matrix. Hence, it is simple and convenient. Second, This approach is applicalbe to control high dimensional hyperchaotic systems by adopting the active control technique, which has the flexiblity to design a control law.

\section{References}

[1] E. Ott, C. Grebogi and J.A. Yorke, "Controlling chaos," Phys. Rev. Lett., vol. 64, pp. 1196-1199, 1990.

[2] L.M. Pecora and T.L. Carroll, "Synchronization in chaotic systems," Phys. Rev. Lett., vol. 64, pp. 821-824, 1990.

[3] G. Perez and H.A. Cerdeira, "Extracting messages masked by chaos," Phys. Rev. Lett., vol. 74 pp. 1970-1973, 1995.

[4] L. Pecora, "Hyperchaos harnessed," World, vol.9, pp. 17-18, 1996.

[5] T.L. Liao and S.H. Tsai, "Adaptive synchronization of chaotic systems and its application to secure communications," Chaos, Solitons \& Fractals, vol. 11, pp. 1387-1396, 2000.

[6] S. Sarasola, F.J. Torredea and A. D'anjou, "Feed-back synchronization of chaotic systems," Int. J. Bifurc. Chaos, vol. 13, pp. 177-191, 2003.

[7] X. Liao, "Chaos synchronization of general Lur'e systems via time-delay feed-back control," Int. J. Bifurc. Chaos, vol. 13, pp. 207-213, 2003.

[8] A. N. Njah and O. D. Sunday, "Synchronization of Identical and Non-identical 4-D Chaotic Systems via Lyapunov Direct Method," Chaos, International Journal of Nonlinear Science, vol. 8, pp. 3-10, 2009.

[9] E.W. Bai and K.E. Lonngren, "Synchronization of two Lorenz systems using active control," Chaos, Solitons \& Fractals, vol. 8, pp. 51-58, 1997.

[10]H.N. Agiza and M.T. Yassen, "Synchronization of Rossler and Chen dynamical systems using active control," Phys. Lett. $A$, vol. 278, pp. 191-197, 2001.

[11] M.T. Yassen, "Chaos Synchronization between two different chaotic system using active control," Chaos, Solitons \& Fractals, vol. 23, pp. 131-140, 2005.

[12] U.E. Vincent, "Synchronization of identical and non-identical 4-D chaotic systems using active control," Chaos, Solitons \& Fractals, vol. 37, pp. 1065-1075, 2008.

[13]R.A. Tang, Y.L. Liu and J.K. Xue, "An extended active control for chaos synchronization," Phys. Lett. A, vol. 373, pp. 1449-1454, 2009.

[14] X.H. Tan, T.Y. Zhang and Y.R. Yang, "Synchronizing chaotic systems using backstepping design," Chaos, Solitons \& Fractals, vol. 16 pp. 37-45, 2003.
[15] J. Zhang, C. Li, H. Zhang and Y. Yu, "Chaos synchronization using single variable feed-back based on backsepping method," Chaos, Solitons \& Fractals, vol. 21, pp. 1183-1193, 2004.

[16] B. Wang and G.J. Wen, "On the synchronization of a class of chaotic systems based on backstepping method," Lett. A, vol. 370, pp. 35-39, 2007.

[17] A.N. Njah, K.S. Ojo, G.A. Adebayo and A.O. Obawole, "Generalized control and synchronization of chaos in RCLshunted Josephson junction using backstepping design," Physica C, vol. 470, pp. 558-564, 2010.

[18]Q. Jia, "Adaptive control and synchronization of a new hyperchaotic system with unknown parameters," Phys. Lett. A, vol. 362, pp. 424-429, 2007.

[19]H. Zhang, X.K. Ma, M. Li and J.L. Zou, "Controlling and tracking hyperchaotic Rossler system via active backstepping design," Chaos Solitons \& Fractals, vol. 26, pp. 353-361, 2005.

[20]M. Jang, C. Chen and C. Chen, "Sliding mode control of hyperchaos in Rossler systems," Chaos Solitons \& Fractals, vol. 14, pp. 1465-1476, 2002.

[21] M. Krstic, I. Kanellakopoulus and P. Kokotovic, Nonlinear and adaptive control design, John Wiley \& sons, Inc., 1995.

[22] G.Y. Qi, M.A. van Wyk, B.J. van Wyk and G.R. Chen, "On a new hyperchaotic system," Phys. Lett. A, vol. 372, pp. 124 136, 2008.

[23] G. Grassi, "Observer-based hyperchaos synchronization in cascaded discrete-time systems," Chaos, Solitons and Fractals, vol. 40, pp. 1029-1039, 2009.

[24] A.Y. Aguilar-Bustos and C. Cruz-Hernández, "Synchronization of discrete-time hyperchaotic systems: an application in communications," Chaos, Solitons and Fractals, vol. 41, pp. 1301-1310, 2009.

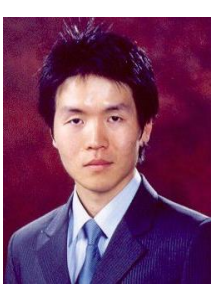

Sung-Hoon $Y u$ received the B.S. and the M.S degrees in electronic engineering from Yonsei University, Seoul, Korea, in 2005 and 2007. He is currently a Ph. D. candidate of Dept. of electrical and electronic engineering in Yonsei University. His current research interests include intelligent control, robust control, adaptive control, chaos system and robot manipulate.

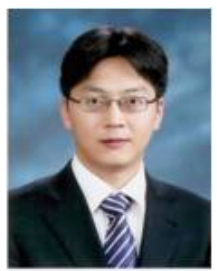

Chang-Ho Hyun received the B.S. degrees in control and instrumentation engineering from Kwangwoon University, Seoul, Korea, and the M.S. and Ph.D. degrees in electrical and electronic engineering from Yonsei University, Seoul, Korea, in 1999. 2002, 
2008. From 2008 to 2009 , he was a senior engineering in Samsung Electronics. Since 2009, he has joined the faculties of the School of Electrical, Electronic and Control Engineering at Kongju National University, where he is currently a full-time lecturer. His current research interests include intelligent control and application, nonlinear control, robotics, mobile robots.

E-mail : hyunch@kongju.ac.kr

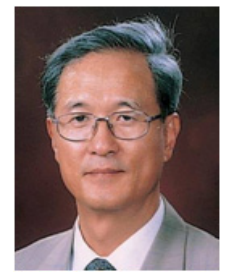

Mignon Park received a B.S. and an M.S.

in electronics from Yonsei University, Seoul, Korea, in 1973 and 1977, and a Ph.D. from the University of Tokyo, Tokyo, Japan, in 1982. He was a researcher in the Institute of Biomedical Engineering, University of Tokyo, from 1972 to 1982 , as well as at the Massachusetts Institute of Technology, Cambridge, and the University of California Berkeley, in 1982. He was a visiting researcher in the Robotics Division, Mechanical Engineering Laboratory Ministry of International Trade and Industry, Tsukuba, Japan, from 1986 to 1987. He has been a Professor in the Department of Electrical and Electronic Engineering, Yonsei University, since 1982. His research interests include fuzzy control and applications, robotics, and fuzzy biomedical systems.

E-mail : mignpark@yonsei.ac.kr 